\title{
Epilepsie
}

\section{Antikonvulsiva erfolgreich kombinieren}

Unter einer Monotherapie mit Antikonvulsiva wird im Schnitt nur jeder zweite Epilepsiepatient langfristig anfallsfrei. Daran hat Professor Bernhard Steinhoff, Epilepsiezentrum Kork, erinnert. Scheitert eine erste Monotherapie, dann stellt sich für die Ärzte oft die Frage, ob sie eine weitere Monotherapie mit einem anderen Medikament versuchen, wie es in bisherigen Empfehlungen favorisiert wird, oder gleich auf eine Kombination setzen sollen. Die Evidenzlage für eine alternative Monotherapie sei nicht besonders gut, so Steinhoff. Solche Empfehlungen beruhten weitgehend auf der Beobachtung, dass es bei einer Kombinationstherapie häufig zu Arzneimittelinteraktionen kommt. Diese ließen sich mit modernen Medikamenten aber oft vermeiden.

Eine gute Kombinierbarkeit wurde etwa für Lacosamid (Vimpat ${ }^{\circledast}$ ) belegt. Das Medikament verstärkt selektiv die langsame, nicht aber die schnelle Inaktivierung von Natriumkanälen. Es wurde unter anderem in drei großen randomi- siert-kontrollierten Studien bei mehr als 1.300 Patienten geprüft, die trotz Antikonvulsiva-Therapie vier oder mehr fokale Anfälle pro Monate hatten. Sie erhielten entweder zusätzlich Lacosamid in Dosierungen von $200-600 \mathrm{mg} / \mathrm{d}$ oder Placebo. In einer gepoolten Analyse dieser Studien kam es bei 23,1 \% der Patienten in den Placebogruppen zur einer Anfallsreduktion um mindestens $50 \%$, bei einer Zusatztherapie mit $200 \mathrm{mg}$ Lacosamid lag der Anteil bei knapp 35\%, mit $400 \mathrm{mg}$ bei $44,3 \%$ und mit $600 \mathrm{mg}$ bei knapp $49 \%$.

Interessant waren die Ergebnisse auch, so Dr. Stephan Arnold, Neurozentrum Nymphenburg München, wenn man unterschied, ob Lacosamid mit klassischen Natriumkanalblockern oder mit anderen Antikonvulsiva kombiniert wurde. Mit klassischen Natriumkanalblockern wie Lamotrigin oder Oxcarbazepin war die Ansprechrate etwas geringer: Hier gelang mit der maximal empfohlenen Lacosamid-Tagesdosis von $400 \mathrm{mg} / \mathrm{d}$ bei $40 \%$ eine Anfallsreduktion um die Hälfte, da- gegen lag dieser Anteil bei einer Lacosamid-Kombination mit anderen Wirkstoffen (etwa Levetiracetam, Valproat, Topiramat) bei $62 \%$. Die Verträglichkeit war unabhängig vom Kombinationspartner gut, am häufigsten wurden Schwindel, Übelkeit und Diplopie beobachtet.

Bestätigt wird die gute Kombinierbarkeit auch durch Ergebnisse einer Interimsanalyse der nicht-interventionellen Studie VITOBA (Vimpat Added To One Baseline Antiepileptic Drug). Die Patienten hatten zunächst trotz Monotherapie weiterhin im Median zwei Anfälle pro Monat. Nach sechs Monaten waren $43 \%$ von 99 Patienten unter einer Kombitherapie mit Lacosamid anfallsfrei, knapp $78 \%$ erzielten eine Anfallsreduktion um mindestens $50 \%$.

Thomas Müller, Springer Medizin

Satellitensymposium „Neue Leitlinien Epilepsie Praxisrelevanz in Fallbeispielen" im Rahmen des 85. DGN-Kongresses, Hamburg, 27.9.12 Veranstalter UCB Pharma

\section{Mehr initiale Therapieoptionen in neuer Leitlinie}

Trotz der noch unzureichenden Datenlage zur möglicherweise neuroprotektiven Wirkung einiger Substanzen zur Behandlung der Parkinson-Erkrankung, ist in jedem Fall eine möglichst frühe Behandlung indiziert, betonten Experten auf einem Symposium beim DGN-Kongress in Hamburg. Referiert wurden wesentliche Neuerungen der aktualisierten Leitlinie zu dieser Erkrankung.

"Zahlreiche klinische und bildgebende Studien“, so Professor Heinz Reichmann, Direktor der Neurologischen Uniklinik in Dresden, „deuten darauf hin, dass die Frühphase der Erkrankung entscheidend für die weitere Progression ist." So zeigt sich bereits 18 Monate nach Diagnosestellung zwischen unbehandelten und mit irgendeinem Parkinson-Medikament in Monotherapie behandelten Patienten ein signifikanter Unterschied in der Lebensqualität [Grosset D et al. J Neurol Neurosurg Psychiatry 2007: 78 (5): 465-
9]. Aus dieser Datenlage zieht die neue Leitlinie den Schluss: „Die pharmakologische Therapie des idiopathischen Parkinson-Syndroms sollte frühzeitig beginnen, effizient und gut verträglich sein.“ Empfohlen wird eine Therapieeinleitung direkt nach der Diagnosestellung, denn dies „scheint den Langzeitkrankheitsverlauf günstig zu beeinflussen“.

Für die Therapieeinleitung gilt eine Differenzierung gemäß dem Alter. Patienten unter 70 Jahre sollten initial mit einem Non-Ergot-Dopamin-Agonisten (DA) behandelt werden; ab dem 70. Lebensjahr kann mit L-Dopa begonnen werden. Reichmann wies jedoch ausdrücklich darauf hin, dass dieser AltersCut-off sehr großzügig zu interpretieren ist. „Entscheidend ist das biologische Alter. So kann bei manchen 75-Jährigen noch mit einem DA begonnen werden."

Ebenso sieht die neue Leitlinie als Alternative vor, bei milder Symptomatik mit einem MAO-B-Hemmer zu starten. Dies ist laut Reichmann sowohl bei jüngeren als auch älteren Menschen mit PD möglich. Die Leitlinie bestätigt die Vorteile von Rasagilin (Azilect ${ }^{\oplus}$ ) gegenüber Selegilin, sagte Reichmann. „Bei Rasagilin wird eine geringere zentralnervöse und kardiovaskuläre Nebenwirkung angenommen."

Die neue Leitlinie weist außerdem auf die gute symptomatische Wirkung des MAO-B- Hemmers hin, welche die OnZeit verlängert. Rasagilin ist in dieser Hinsicht demnach mit der Wirkung des COMT-Hemmers Entacapon vergleichbar und kann mithin auch zu dopaminergen Präparaten hinzukombiniert werden.

Reimund Freye, freier Medizinjournalist

Satellitensymposium: „Die unterschiedlichen Herausforderungen in der Behandlung des M. Parkinson", 85. DGN-Kongress, Hamburg, 27.9.2012; Veranstalter: TEVA 